\title{
Satisfacción del usuario acerca del servicio de transporte público de pasajeros
}

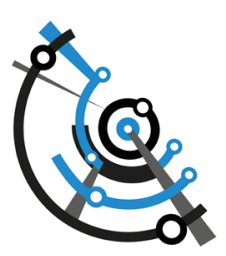

\section{Javier Romero-Torres}

Área de Ingeniería en Transporte, Centro Universitario Nezahualcóyotl, Universidad Autónoma del Estado de México, México.

https://orcid.org/oooo-0001-9830-7630

\section{Javier García Gutiérrez}

Área de Posgrado, Faculta de Ingeniería, Universidad Autónoma del Estado de México, México.

https://orcid.org/oooo-0003-2654-5271

\section{Noé Gaspar Sánchez}

Área de Ingeniería en Transporte, Centro Universitario Nezahualcóyotl, Universidad Autónoma del Estado de México, México. https://orcid.org/oooo-0003-4597-8865

Recibido: 24 de diciembre de 2018. Aceptado: 20 de agosto de 2019.

\begin{abstract}
Resumen
En este trabajo se reporta las derivaciones de la valoración de la satisfacción del usuario acerca del servicio de transporte de autobuses para la zona de la ciudad de Toluca, México. Los factores identificados en una primera fase fueron tres cuantitativos: tiempo de espera (te), tiempo a bordo del autobús (tv), y costo del viaje (cv): tarifa; y 15 factores cualitativos enmarcados en tres dimensiones del servicio: forma de manejar (foma), trato que recibe el usuario (trato) y estado físico del autobús (edofís). Posteriormente se diseñó y aplicó una encuesta de satisfacción para obtener las percepciones de los usuarios acerca de los niveles de calidad de los 18 factores previamente identificados, esas percepciones se lograron atrapar mediante escalas tipo Likert con cinco niveles previamente seleccionadas y afinadas mediante un grupo focal. A partir del análisis realizado se infiere que para aumentar la satisfacción del usuario es necesario llevar a cabo implementaciones dirigidos a factores cualitativos más que en los aspectos operativos. Lo que permitirá a la empresa de transporte público redefinir y administrar sus directrices de calidad de servicio con la finalidad de mejorar su imagen, repercutiendo en una mejoría del usuario durante el viaje en transporte público.
\end{abstract}




\title{
User satisfaction about the public passenger transport service
}

\begin{abstract}
This paper reports the derivations of the assessment of user satisfaction about the bus transport service for the area of the city of Toluca, Mexico. The factors identified in a first phase were three quantitative: waiting time, time on board the bus, and travel cost: fare; and 15 qualitative factors framed in three dimensions of the service: driving style, treatment that the user receives and physical condition of the bus. Afterwards, a satisfaction survey was designed and applied to obtain the users' perceptions about the quality levels of the 18 previously identified factors. These perceptions were captured using Likert scales with five levels previously selected and refined by a focus group. Based on the analysis carried out, it is inferred that in order to increase user satisfaction, it is necessary to carry out implementations aimed at qualitative factors rather than operational aspects. This will allow the public transport company to redefine and manage its quality of service guidelines in order to improve its image, resulting in an improvement of the user during the trip in public transport.
\end{abstract}

Keywords: User satisfaction. Public transport. Toluca-Mexico.

Palavras-chave: Satisfação do usuario. Transporte público. Toluca-México.

\section{Introducción}

La satisfacción del cliente, usuario para el caso de transporte público de pasajeros (TP), como medida de calidad se ha introducido en la investigación de mercado. En este campo, existe un amplio abanico de técnicas para calcular la satisfacción del cliente. Una técnica más conocida y con frecuencia más aplicada es el método ServQual, propuesto por Parasuraman, et al. (1985). El método ServQual introdujo el concepto de satisfacción del cliente como una función de las expectativas, las cuales indican lo que el usuario requiere del servicio y las percepciones que son los niveles que el usuario recibe, dicho método fue elaborado para medir la satisfacción en organizaciones (Gilbert y Veloutsou, 2006), pero con la desventaja en cuanto al límite de dimensiones y factores a utilizar.

Algunas variaciones de este método se introdujeron en años posteriores como el método ServPerf, la Normed Quality (NQ), el índice de satisfacción confiable, el barómetro sueco de satisfacción del cliente (SCSB), el índice de satisfacción del cliente estadounidense (ACSI), el barómetro noruego de satisfacción del cliente (NCSB) o el Índice Europeo de Satisfacción del Cliente (ECSI); métodos que en general han estandarizado su aplicación para cada zona o población objetivo, para más detalle de esto consultar Eboli y Mazzulla (2009). Dentro del estudio de la satisfacción del usuario para el transporte público se puede estimar utilizando distintos enfoques, uno de ellos se refiere a calcular el desempeño de los atributos o bien utilizando el desempeño y las expectativas.

En el mismo contexto podemos encontrar modelos agregados los cuales utilizan un análisis de los atributos del servicio para obtener satisfacción total del servicio; por otro lado, existen los modelos desagregados con los cuales obtenemos un análisis por separado de los diferentes atributos del servicio. Para el lector interesado en ahondar en los enfoques metodológicos para la medición de la satisfacción puede consultar de Oña y de Oña (2014) o Grigoroudis y Siskos (2010).

Este trabajo tiene dos objetivos: a) proponer una caracterización de la satisfacción a partir del usuario, incorporando elementos cuantitativos (factores duros) y cualitativos 
(elementos suaves) mediante una agregación de factores y subfactores; $y \mathrm{~b}$ ) exponer los niveles y escalas de medición de los factores asociados a la satisfacción; estas escalas incluyen una etiqueta y valor número en cada nivel. Con lo anterior, se presenta una forma de análisis de las percepciones acerca de los niveles de satisfacción por parte de los usuarios de transporte de pasajeros para un caso de estudio.

Este artículo está organizado en la siguiente manera: en la sección dos se describe la zona y el sistema de TP considerado en este estudio; en la sección tres se expone la definición y cómo medir la satisfacción; en la sección cuatro se ilustran las características del usuario y del viaje en TP reportado, se describen los resultados acompañados de las conclusiones.

\section{Caso de estudio}

El sistema de transporte público de autobuses de la ciudad mexicana de Toluca está comprendido por 190 rutas ofertadas por 15 empresas de trasporte urbanos y suburbano; aproximadamente el parque vehicular es de 2,600 unidades (autobuses). En 2007 se estimó que se realizaban 795 mil viajes al día, con un promedio de 72 mil viajes en el período de máxima demanda (06:45-07:45h) y 460 pasajeros promedio por unidad al día, ver Cuadro 1 .

Cuadro 1. Empresas de transporte público de autobuses. Fuente: Hinojosa et al. (2014).

\begin{tabular}{|l|c|c|}
\hline Empresa & Rutas & $\begin{array}{c}\text { Parque } \\
\text { vehicular }\end{array}$ \\
\hline RED DE TRANSPORTE PÚBLICO S.A. DE C.V. & 22 & 354 \\
\hline SERVICIO INTERMETROPOLITANO DE TRANSPORTE S.A. DE C.V. & 19 & 338 \\
\hline SERVICIOS URBANOS Y SUBURBANOS XINANTECATL S.A. DE C.V. & 20 & 261 \\
\hline $\begin{array}{l}\text { SISTEMA DE TRANSPORTE URBANO Y SUBURBANO DE LA CIUDAD DE } \\
\text { TOLUCA S.A. DE C.V. }\end{array}$ & 14 & 289 \\
\hline $\begin{array}{l}\text { AUTOTRANSPORTES URBANOS Y ZONA CONURBANDA DEL VALLE DE } \\
\text { TOLUCA ADOLFO LÓPEZ MATEOS S.A. DE C.V. }\end{array}$ & 13 & 236 \\
\hline $\begin{array}{l}\text { AUTOTRANSPORTES URBANOS Y SUBURBANOS TOLLOTZIN S.A. DE } \\
\text { C.V. }\end{array}$ & 9 & 89 \\
\hline $\begin{array}{l}\text { AUTOTRANSPORTES URBANOS DE TOLUCA Y ZONA CONURBADA } \\
\text { S.A. DE C.V. }\end{array}$ & 5 & 79 \\
\hline TRANSPORTES URBANOS Y SUBURBANOS TOLLOCAN S.A. DE C.V. & 10 & 142 \\
\hline $\begin{array}{l}\text { AUTOBUSES MÉXICO TOLUCA ZINACANTEPEC Y RAMALES S.A. DE } \\
\text { C.V. }\end{array}$ & 4 & 36 \\
\hline $\begin{array}{l}\text { AUTOTRANSPORTES TOLUCA CAPULTITLÁN TRIANGULO ROJO S.A. } \\
\text { DE C.V. }\end{array}$ & 20 & 108 \\
\hline $\begin{array}{l}\text { AUTOBUSES FLECHA BLANCA DE TOLUCA S.A. DE C.V. } \\
\text { AUTOTRANSPORTES URBANOS Y SUBURBANOS DE LA CIUDAD DE } \\
\text { TOLUCA Y ZONA INDUSTRIAL S.A. DE C.V. (ATZUSI) }\end{array}$ & 16 & 180 \\
\hline $\begin{array}{l}\text { LÍNEA DE TURISMO TOLUCA - TENANGO - ESTRELLA DE ORO S.A. DE } \\
\text { C.V. }\end{array}$ & 5 & 43 \\
\hline AUTOTRANSPORTES COLÓN NACIONAL S.A. DE C.V. & 15 & 278 \\
\hline \begin{tabular}{l} 
AUTOTRANSPORTES 8 DE NOVIEMBRE S. DE R.L. DE C.V. \\
\hline Total
\end{tabular} & 190 \\
\hline
\end{tabular}


La cobertura del sistema de transporte público de autobuses es aceptable, ya que abarca un alto porcentaje de la estructura urbana actual. Una particularidad de este sistema es el problema de sobreoferta, sin embargo, este rasgo no es garantía de la calidad de servicio (CS), ya que al no existir una planeación en los tres niveles de decisión (van de Velde, 1999): estratégico, táctico y operativo existen deficiencias, algunos de estas negatividades son (Hinojosa et al., 2014):

" Una red de transporte desarticulada y un excesivo número de unidades.

» Tasas bajas de ocupación (35\%), lo cual manifiesta un exceso de oferta y baja rentabilidad de las rutas.

"Sobreposición de rutas (80\%) en avenidas principales de la ciudad.

" Tiempos de viaje excesivos con velocidades bajas (en promedio 2 horas con 15 minutos para cubrir una ruta).

»Sinuosidad de las rutas de 1,7, lo que implica un recorrido excesivo más para llegar al destino.

» Unidades en malas condiciones físicas y mecánicas (40\% de las unidades tienen una antigüedad superior a 10 años).

》 Niveles deficientes de seguridad para los pasajeros.

"Irresponsabilidad de los conductores al conducir las unidades.

» No respeto a las señales de tránsito.

\section{Definición y escalas de medición}

Para obtener los juicios de las usuarios acerca de la satisfacción del servicio se diseñó una encuesta, la cual estuvo integrada por tres secciones (Stopher, 2012), en la primera se incorporaron elementos de control y manejo de los datos como la fecha, hora, punto de aplicación, nombre del encuestador; la segunda sección permitió obtener datos referentes al viaje (e.g. frecuencia, motivo del desplazamiento) así como algunos datos socioeconómicos del usuario (e.g. edad, sexo, ingreso mensual). La tercera parte estuvo integrada por los cuestionamientos para determinar el grado de satisfacción del servicio en relación a los niveles de los elementos cuantitativos; tiempo de espera (te), tiempo de viaje a bordo del autobús (tv ), costo del viaje (cv), y tres factores cualitativos: forma de manejar del conductor (foma), el trato al usuario por parte del conductor (trato) y el estado físico del autobús (edofís), estos tres últimos definidos por 15 subfactores/ especificaciones (ver Cuadro 2). 
Cuadro 2. Factores y especificaciones utilizadas en la encuesta de satisfacción. Fuente: Elaboración propia.

\begin{tabular}{|c|c|c|}
\hline Factor & Especificación & Etiqueta \\
\hline \multirow[t]{5}{*}{ Foma } & Detiene completamente el autobús para bajar y subir a los pasajeros & detbus \\
\hline & Conduce a una velocidad adecuada & velade \\
\hline & Respeta los señalamientos viales y de tránsito (y luces del semáforo) & resseñ \\
\hline & Va concentrado en el manejo del autobús & conman \\
\hline & Se salió de la ruta & salrut \\
\hline \multirow[t]{5}{*}{ Trato } & Utiliza un vocabulario adecuado (decente) con usted & vocade \\
\hline & Lo trata de manera respetuosa & trares \\
\hline & Es honesto en el cobro de la tarifa (y le da su cambio) & honcob \\
\hline & Es amable & amable \\
\hline & Tiene higiene personal y presentación (vestimenta) adecuadas & higper \\
\hline \multirow[t]{5}{*}{ Edofís } & $\begin{array}{l}\text { El pasamanos, tubos, ventanas, puertas, timbre funcionan } \\
\text { adecuadamente }\end{array}$ & pasman \\
\hline & Adecuada iluminación interior & iluint \\
\hline & El espacio (dimensiones) de los asientos es adecuado & espasi \\
\hline & Los asientos están en condiciones adecuadas & asiade \\
\hline & Existe visibilidad en las ventanas & visven \\
\hline $\begin{array}{l}\text { Tiempo de } \\
\text { espera }\end{array}$ & & te \\
\hline $\begin{array}{l}\text { Tiempo de } \\
\text { viaje } \\
\text { (a bordo } \\
\text { del } \\
\text { autobús) }\end{array}$ & & tv \\
\hline $\begin{array}{l}\text { Costo del } \\
\text { viaje }\end{array}$ & & $\mathrm{CV}$ \\
\hline
\end{tabular}

En la actualidad, es necesario realizar mediciones para hacer evaluaciones y predicciones de productos o servicios, la satisfacción en el transporte público de autobuses es uno de esos temas; en relación a ella es posible obtener información utilizando un comportamiento (elegir) u obtener un juicio (qué piensa el usuario del servicio). En los sistemas de transporte, la elección es la medida más popular para revelar la satisfacción o CS como una utilidad, mientras los juicios son mediciones que son ampliamente preferidos en estudios de mercado. En este caso, se utilizan escalas numéricas, escalas de descripción verbal o escalas de punto medio (Grigoroudis y Siskos, 2010) o (Stopher, 2012). Una de la escala más común para medir la satisfacción del usuario de TP (o aspectos relacionados con la salud, creencias o comportamientos) es la denomina Likert. Éstas ostentan las siguientes estructuras (Losby y Wetmore, 2012):

¿ Establece sentencias declarativas

"Categorías ordenadas de respuesta

" Número balanceado de opciones positivas y negativas

"Descripción de cada categoría

» Valor numérico asignado a cada categoría

Para determinar las escalas utilizadas en el diseño de la encuesta de satisfacción se consideró una metodología establecida (Elejabarrieta e Iñiguez, 1984). La validación de cada escala utilizada en la encuesta de satisfacción fue apoyada mediante grupos focales y encuestas piloto; una revisión teórica acerca de las escalas para medir calidad o satisfacción del usuario en TP está expuesta en Romero et al. 
(2016) referente a un sistema de trasporte de pasajeros. Las escalas del tiempo de espera (te) y el tiempo de viaje (tv) están ligada a una percepción de la satisfacción referida a la cantidad de minutos empleados; la escala para la tarifa (cv) estuvo ligada a lo barato o costoso de ella; la escala para la forma de manejar (foma) estuvo relacionada a una percepción de la seguridad referente a la integridad física del usuario como el sufrir algún accidente a bordo del autobús; la escala para el trato al usuario (trato) está determinada por una percepción en relación a la atención recibida; y la escala del estado físico del autobús (edofís) está ligada tanto a una escala de seguridad como a una escala de comodidad. Las escalas utilizadas en la encuesta se visualizan en el Cuadro 3.

Cuadro 3. Escalas para valorar la satisfacción. Fuente: Elaboración propia.

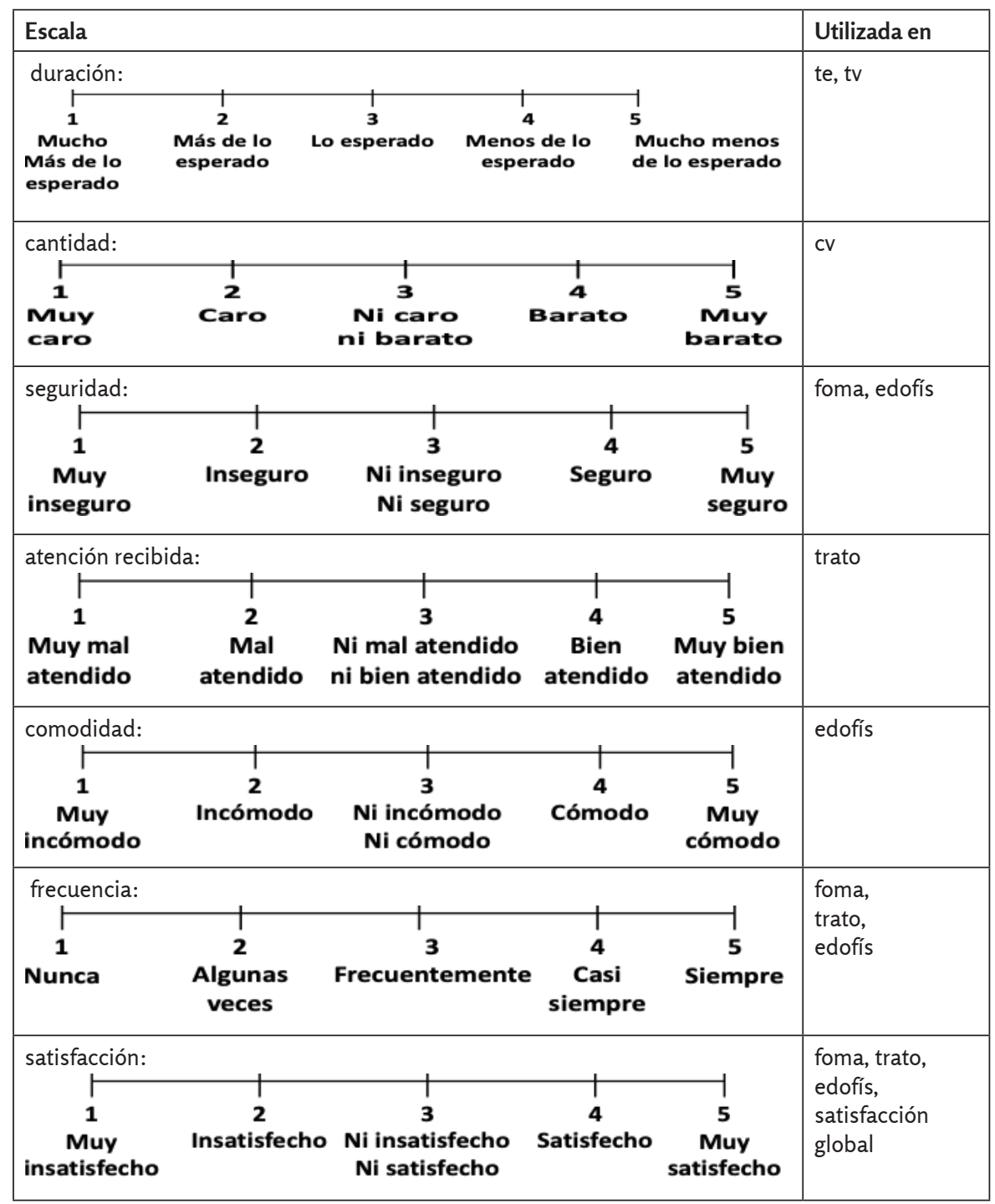

\section{Resultados y conclusiones}

Se aplicaron 332 encuestas repartidas en siete puntos de la zona de estudio, debido al enfoque exploratorio del estudio, la muestra fue no representativa, el muestreo fue incidental considerando que los participantes fueran usuarios del transporte de autobuses. 
Lo anterior se logró al interceptar a los usuarios en la etapa de trasbordo, los lugares de aplicación corresponden a los puntos principales de intercambio modal de la zona de estudio. Los datos de esta encuesta se observan en los Cuadros 4 y 5.

En promedio los usuarios del TP en la zona de la ciudad de Toluca viajan 45 minutos, erogando 22 pesos mexicanos (MXN), actualmente la tarifa urbana mínima es de 10 MXN, lo que implica que la otra parte del costo de transporte corresponde al tramo del viaje suburbano; y en promedio esperan casi 13 minutos en las paradas. Este periodo prolongado de espera es contrario al $80 \%$ de sobreposión de las rutas, sin embargo se debe tener presente que los usuarios deben esperar específicamente aquel autobús que los lleve a su destino.

Cuadro 4. Características del viaje. Fuente: elaboración propia.

\begin{tabular}{|l|c|c|c|c|c|}
\hline Punto de trasbordo & $\begin{array}{c}\text { Encuestas } \\
\text { aplicadas }\end{array}$ & $\%$ & $\begin{array}{c}\text { cv } \\
(\mathbf{M X N})\end{array}$ & $\begin{array}{c}\text { tv } \\
(\mathbf{m i n})\end{array}$ & $\begin{array}{c}\text { te } \\
(\mathbf{m i n})\end{array}$ \\
\hline Chedraui & 50 & 15,1 & 16,5 & 46,3 & 10,5 \\
\hline Sendero & 48 & 14,5 & 9,3 & 42,8 & 14,3 \\
\hline Walmart & 50 & 15,1 & 19,3 & 49,2 & 14,9 \\
\hline Pino Suárez & 44 & 13,3 & 43,5 & 49,4 & 12,7 \\
\hline Colón & 50 & 15,1 & 28,1 & 52,9 & 14,1 \\
\hline Cosmovitral & 50 & 15,1 & 8,9 & 37,8 & 12,6 \\
\hline ExCama Piedra & 40 & 12,0 & 34,6 & 42,2 & 10,5 \\
\hline Total & 332 & 100 & -- & -- & -- \\
\hline Promedio & -- & -- & 22,3 & 45,9 & 12,9 \\
\hline
\end{tabular}

La proporción de las encuestas aplicadas de acuerdo al género es adecuada. Una quinta parte de los entrevistados indicaron no tener ingresos, generalmente este estrato corresponde a estudiantes y madres de familia dedicadas al trabajo de la casa, el 36,7\% usuarios del TP son de ingresos bajos, aceptando recibir mensualmente hasta 3.000 MXN $(1 \mathrm{USD}=19,30 \mathrm{MXN})$, y el 5,7\% indicó un ingreso mayor a 8,000 MXN; esto indica que la proporción mayoritaria de usuarios son de ingresos bajos. El 84\% de los entrevistados ostentan una edad de 18 a 50 años, representando la población económicamente activa o estudiantes universitarios, lo anterior reforzado por las dos terceras partes de los usuarios $(62,3 \%)$ del TP que se desplazan por razones de trabajo o escuela; en segundo término ir de compras y visitas representan 22,6\% de los desplazamientos. Mientras viajeros cotidianos ( $>3$ viajes/semana) representan el $66.3 \%$. Es decir, la radiografía de los usuarios de TP indica que éstos son estratos de la población de bajos ingresos, en edad laboral, con desplazamientos obligados, y viajeros cotidianos, lo que se percibe como usuarios cautivos.

Cuadro 5. Características del usuario. Fuente: Elaboración propia.

\begin{tabular}{|c|c|c|c|c|c|c|c|c|c|}
\hline $\begin{array}{l}\text { Viajes/ } \\
\text { semana }\end{array}$ & $\%$ & Motivo & $\%$ & $\begin{array}{l}\text { Edad } \\
\text { (años) }\end{array}$ & $\%$ & $\begin{array}{l}\text { Ingreso } \\
\text { mensual(\$) }\end{array}$ & $\%$ & Sexo & $\%$ \\
\hline 1 & 9,3 & Trabajo & 41,3 & $0-18$ & 6,3 & Sin ingreso & 19,9 & Mujer & 52,4 \\
\hline 2 & 13,6 & Escuela & 21,1 & $19-30$ & 35,8 & $100-1.500$ & 19,3 & Hombre & 47,6 \\
\hline 3 & 10,8 & Compras & 13,0 & $31-40$ & 27,7 & $1.501-3.000$ & 17,5 & & \\
\hline 4 & 4,2 & Visita & 9,6 & $41-50$ & 20,5 & $3.001-4.500$ & 16,6 & & \\
\hline 5 & 36,7 & Negocios & 2,7 & $51-60$ & 5,1 & 4.501-6.000 & 12,3 & & \\
\hline 6 & 19,9 & Diversión & 1,5 & $>60$ & 4,5 & $6.001-8.000$ & 8,7 & & \\
\hline 7 & 5,4 & Trámites & 3,3 & & & $8.001-12.000$ & 3,9 & & \\
\hline & & Otro & 7,5 & & & $>12.000$ & 1,8 & & \\
\hline
\end{tabular}


Para el tiempo de espera (te), la más alta valoración, con un promedio (Prom.) de 3,0 , fue identificada en el intervalo de 3,1 a 5 minutos, estableciendo que el rango de espera en el sistema de autobuses del caso es estudio debe ser como máximo cinco minutos. En general, las mejores valoraciones se observaron en valores de tiempo de hasta 15 minutos, las más bajas para tiempos de espera mayores, ver Cuadro 6. La valoración promedio de la duración del te fue de 2,4, este valor se encuentra comparado con los promedios (Prom.) para cada intervalo del tiempo de espera en la Figura 1. Los tiempos de espera son mayores a lo esperado por los usuarios, percepción que prevalece en todos los puntos de transferencia modal; que si bien la valoración de este factor se degrada conforme aumenta la espera, también es cierto que con valores relativamente bajos ( 3 a 5 minutos de espera) se observan valoraciones pobres. Los valores máximos de satisfacción se observan para esperas de cinco minutos lo que se traduce en intervalos de paso de 10 minutos, que es un valor similar $(\approx 11$ minutos) al que actualmente están operando las rutas de transporte de pasajeros de la zona de estudio (UAEM-GEM, 2016), es decir, una frecuencia actual de 6 autobuses en una hora propicia una mayor satisfacción.

Cuadro 6. Satisfacción en relación al te. Fuente: Elaboración propia.

\begin{tabular}{|l|c|c|c|c|c|c|}
\hline \multirow{2}{*}{ te (min) } & \multicolumn{7}{|c|}{ Nivel de satisfacción } & \multirow{2}{*}{ Prom. } \\
\cline { 2 - 7 } & $\mathbf{1}$ & $\mathbf{2}$ & $\mathbf{3}$ & $\mathbf{4}$ & 5 & \\
\hline$<=3$ & & 2 & 3 & & 0 & 2,6 \\
\hline $3,1-5$ & 1 & 5 & 42 & 5 & 0 & 3,0 \\
\hline $5,1-10$ & 15 & 33 & 73 & 8 & 0 & 2,6 \\
\hline $10,1-15$ & 7 & 43 & 24 & 2 & 0 & 2,3 \\
\hline $15,1-20$ & 15 & 19 & 8 & 2 & 0 & 1,9 \\
\hline$>20$ & 14 & 5 & 5 & 1 & 0 & 1,7 \\
\hline
\end{tabular}

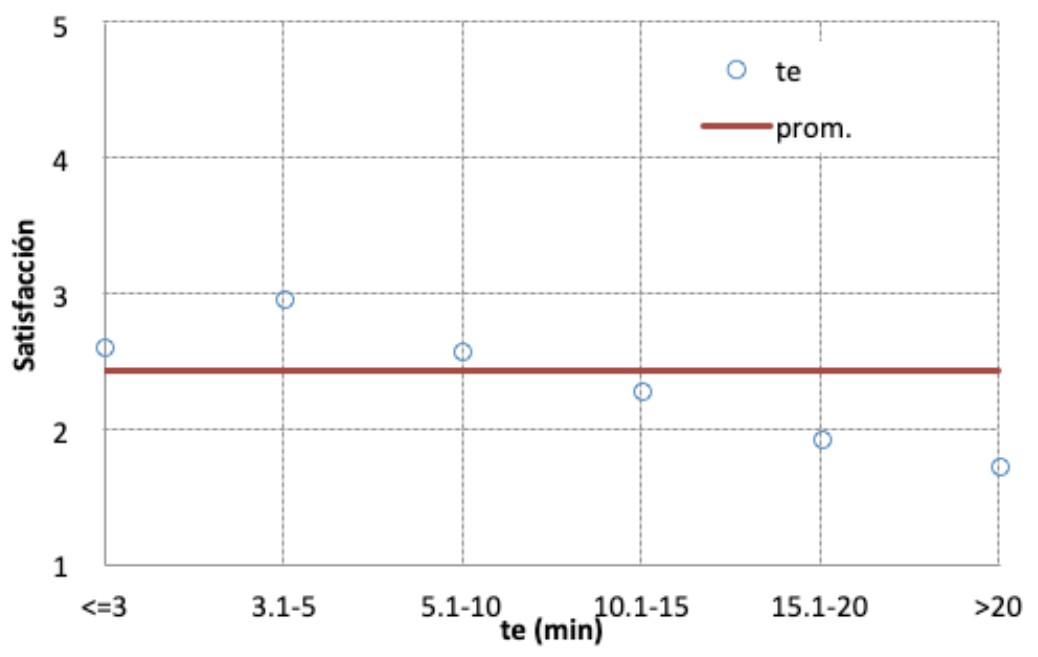

Figura 1. Satisfacción en relación al te. Fuente: Elaboración propia.

El promedio de la valoración de la duración del tiempo de viaje (tv) fue 2,4; la más favorable fue emitido por aquellos entrevistados que aceptaron invertir hasta quince minutos en sus traslados (viajes cortos), sin embargo, valoraciones similares ( 2,5 y 2,6$)$ fueron expresadas para intervalos de tiempo de 15,1 a 30 minutos y para valores de más de una hora de viaje. Lo anterior no es un resultado contraintuitivo si se considera que aquellos viajeros que emplean más de una hora de viaje provienen de zonas de la periferia de la ciudad, es decir, en el análisis se debe considerar la distancia y la manera en que se realiza 
los desplazamientos (e.g. cantidad de trasbordos y modos utilizados). Por otro lado, la valoración más baja $(1,8)$ se identificó en el intervalo de 45,1 a 60 minutos, encontrándose en este período el promedio de 45,9 minutos (ver cuadro 4), es decir esta valoración es representativa de todo el sistema de autobuses. Aunque las distancias que se recorren en la zona urbana son relativamente cortas es demasiado el tiempo para completarlas en transporte público, por ejemplo para una viaje de 6 kilómetros se ha encontrado que el traslado en autobús puede durar de 45 a 80 minutos. Para más detalle ver el Cuadro 7 y la Figura 2; por otro lado, los tiempos de viaje no únicamente están influenciados por las trayectorias de la ruta sino por niveles de congestión en las vialidades, por lo que una mejora de la satisfacción respecto al tiempo de viaje considerando sólo el sistema de transporte implicaría adecuar el trazo de la rutas con la finalidad de disminuir la sinuosidad.

Cuadro 7. Satisfacción en relación al tv. Fuente: Elaboración propia.

\begin{tabular}{|c|c|c|c|c|c|c|}
\hline \multirow{2}{*}{ tv (min) } & \multicolumn{7}{|c|}{ Nivel de satisfacción } & \multirow{2}{*}{ Prom. } \\
\cline { 2 - 7 } & $\mathbf{1}$ & $\mathbf{2}$ & $\mathbf{3}$ & $\mathbf{4}$ & 5 & 2.9 \\
\hline$<=15$ & 2 & 5 & 9 & 7 & & 2.6 \\
\hline $15.1-30$ & 5 & 44 & 50 & 11 & & 2.3 \\
\hline $30.1-45$ & 13 & 41 & 27 & 4 & & 1.8 \\
\hline $45.1-60$ & 30 & 26 & 12 & 1 & & 2.6 \\
\hline $60.1-90$ & 4 & 10 & 10 & 5 & & 2.5 \\
\hline$>90$ & 3 & 5 & 4 & 3 & & 2 \\
\hline
\end{tabular}

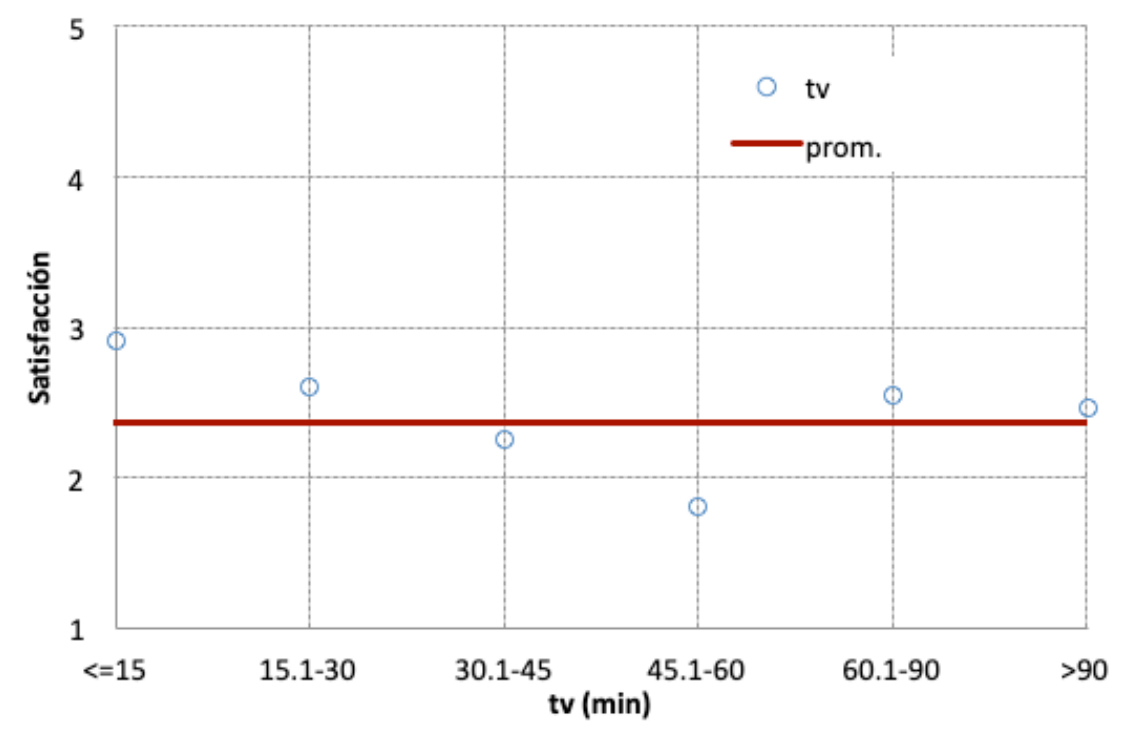

Figura 2. Satisfacción en relación al tv. Fuente: Elaboración propia.

La mejor valoración $(2,6)$ en cuanto a la tarifa en el transporte de autobuses se identificó en el rango de hasta ocho pesos (tarifa mínima en la zona de estudio); la peor valoración $(2,0)$ del cv fue establecida para aquellos usuarios que erogan entre 10,1 y $15 \mathrm{MXN}$, ver Cuadro 8. La valoración promedio fue de 2,4, la interpretación de estos resultados deben estar ligados a la distancia o las zonas entre las cuales se realiza el viaje, ya que en el área de la ciudad de Toluca, para realizar el mismo viaje pueden existir más de una opción, una combinación de cadenas de viaje en que las etapas corresponden a autobuses de distintas rutas y que el cv del viaje es el total de tarifas erogadas en cada eslabón. Por lo que el usuario enfrenta dos escenarios a elegir: a) un viaje sin trasbordos implicando el pago de una tarifa, pero debido a la sinuosidad de la ruta implica un tiempo de viaje abordo 
mayor a lo esperado; $\mathrm{y}$ b) un viaje con transbordos de tal manera que el desplazamiento sea más directo, erogando más de un pago a cambio de un tiempo de viaje menor. En ese tenor al inicio del presente siglo el 53.8\% de los usuarios en la zona conurbada de Toluca realizaban de uno a dos trasbordos (Cárdenas, 2001), situación que no ha cambiado ya que la proporción de usuarios que hacen uno o dos trasbordos se ha mantenido recientemente (Romero et al. 2017). En el Cuadro 8 y Figura 3 se visualiza que las peores percepciones de los usuarios (2,0 y 2,3) corresponden a tarifas de 10 a 20 MXN. Mientras las mejores evaluaciones ( 2,6 y 2,5 , respectivamente) corresponden a las tarifas extremas de 8 y $30 \mathrm{MXN}$; la primera corresponden a aquellos usuarios que completan su viaje con un único pago, generalmente corresponde a viajes dentro de la zona urbana, mientras la segunda está integrada por erogaciones ligadas a una combinación de viajes urbanos y semiurbanos; opuestamente los que pagan de 10 a 15 MXN están de alguna manera obligados a pagar dos tarifas debido a la necesidad de realizar un trasbordo dentro de la zona urbana, lo que sustenta sus valores bajos de la satisfacción referente al costo del viaje. Este trasbordo obligado es con la finalidad de disminuir el tiempo de viaje excesivo que parece estar relacionado a la sinuosidad de la ruta.

Cuadro 8. Satisfacción en relación al cv. Fuente: Elaboración propia.

\begin{tabular}{|l|c|c|c|c|c|c|}
\hline \multirow{2}{*}{ cv (MXN) } & \multicolumn{7}{|c|}{ Nivel de satisfacción } & \multirow{2}{*}{ Prom. } \\
\cline { 2 - 7 } & $\mathbf{1}$ & $\mathbf{2}$ & $\mathbf{3}$ & $\mathbf{4}$ & $\mathbf{5}$ & \multirow{2}{*}{2,6} \\
\hline$<=8$ & 9 & 43 & 91 & 5 & 2,4 \\
\hline $8,1-10$ & 4 & 13 & 11 & 2 & & 2,0 \\
\hline $10,1-15$ & 9 & 7 & 6 & 2 & & 2,3 \\
\hline $15,1-20$ & 8 & 15 & 13 & 3 & & 2,5 \\
\hline $20,1-30$ & 2 & 11 & 9 & 2 & & 2,3 \\
\hline$>30$ & 12 & 26 & 23 & 6 & & 2 \\
\hline
\end{tabular}

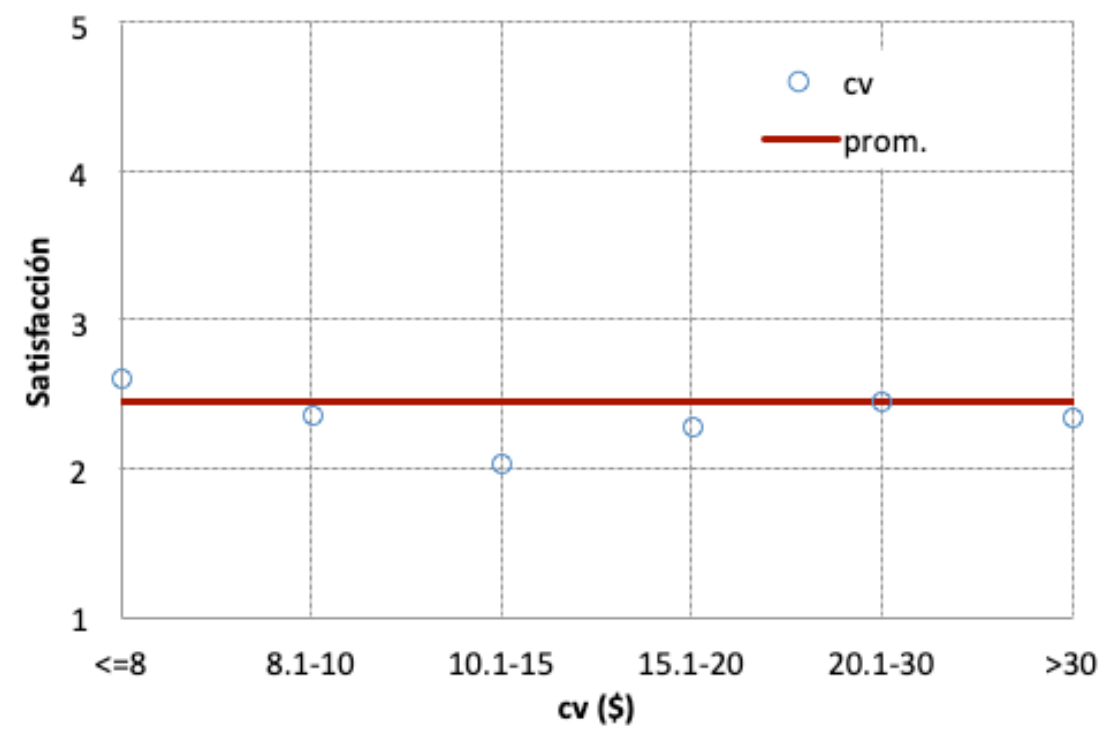

Figura 3. Satisfacción en relación al cv. Fuente: Elaboración propia.

El análisis realizado para el factor cualitativo foma (igualmente para trato y edofís) fue inicialmente calcular la valoración promedio (Prom. total) de cada especificación de acuerdo a los cinco niveles de la satisfacción del factor; posteriormente se obtuvo el cociente de la satisfacción promedio y el promedio total /satisfacción prom./Prom. total), este valor permitió comparar el grado de aportación que cada especificación añade a la satisfacción del 
factor. Continuando con lo anterior, en el Cuadro 9 se muestra lo anterior descrito para el factor foma, algunas interpretaciones son las siguientes. A mayor valor de la satisfacción de foma mayor es el promedio de la satisfacción (Prom. total), similar comportamiento fue presentado para la satisfacción promedio (Satisfacción prom.) de cada especificación, por ejemplo, para la especificación "conduce a una velocidad adecuada" (velade) el promedio de la valoración (vista con la escala de seguridad) fue de 1,5 para la categoría 1 ("muy insatisfecho") de la satisfacción de foma, 2.06 para la categoría 2 ("insatisfecho"), y de 2,7, 3,44 y 3,82 , respectivamente, para las categorías 3,4 y 5 de la satisfacción existe una relación positiva entre las valoraciones de las especificaciones y la satisfacción de foma. En la Figura 4 y Cuadro 9 se observa que para el menor valoración de la satisfacción de foma, la especificación salrut fue $31 \%$ mejor valorada con respecto al promedio, le siguió detbus con el $16 \%$, mientras velade estuvo $20 \%$ por debajo del valor promedio; mientras que para la valoración más alta "muy satisfecho" de foma, salrut fue la especificación que más aportó a esa valoración, con un 10\%. En general, la especificación salrut fue la especificación que más aportó a la satisfacción de foma, y velade la que menos aportó, ver Figura 4.

Cuadro 9. Satisfacción en relación a foma. Fuente: Elaboración propia.

\begin{tabular}{|l|c|c|c|c|c|c|}
\hline $\begin{array}{l}\text { Nivel de } \\
\text { satisfacción }\end{array}$ & \multicolumn{1}{l|l}{ detbus } & \multicolumn{1}{l}{ velade } & \multicolumn{2}{l}{ conman } & salrut & Prom. total \\
\hline 1 & 2,18 & 1,5 & 1,64 & 1,64 & 2,46 & 1,89 \\
\hline 2 & 2,14 & 2,06 & 2,23 & 2,05 & 2,92 & 2,28 \\
\hline 3 & 2,86 & 2,7 & 2,72 & 2,71 & 3,3 & 2,86 \\
\hline 4 & 3,68 & 3,44 & 3,65 & 3,14 & 3,79 & 3,54 \\
\hline 5 & 4,18 & 3,82 & 4,09 & 4 & 4,55 & 4,13 \\
\hline Satisfacción prom./Prom. total & 1,16 & 0,8 & 0,87 & 0,87 & 1,31 & 1 \\
\hline 1 & 0,94 & 0,9 & 0,98 & 0,9 & 1,28 & 1,00 \\
\hline 2 & 1 & 0,94 & 0,95 & 0,95 & 1,15 & 1,00 \\
\hline 3 & 1,04 & 0,97 & 1,03 & 0,89 & 1,07 & 1,00 \\
\hline 4 & 1,01 & 0,93 & 0,99 & 0,97 & 1,1 & 1,00 \\
\hline 5
\end{tabular}

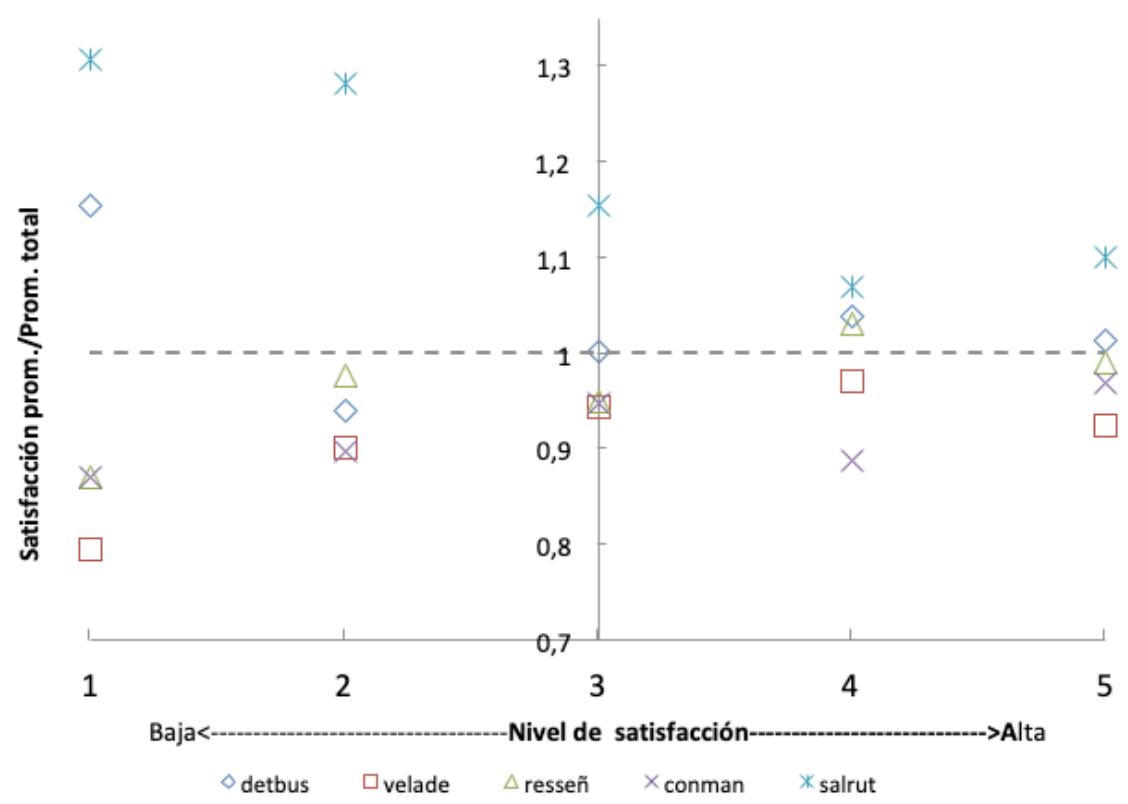

Figura 4. Satisfacción en relación a foma. Fuente: Elaboración propia. 
Para el factor trato, la especificación amable aportó 20\% sobre el promedio para la satisfacción 1, en general la especificación honcob fue la que contribuyó más en la valoración positiva de la satisfacción del trato (con valores del 9 y 10\%). Mientras la especificación amable, seguido de la especificación vocade que aportó valoraciones por arriba del promedio entre 1 y $4 \%$. Por el contrario, la especificación que aportó menos en la satisfacción de foma, con valores del 4 y $5 \%$ por debajo del promedio, fue amable, para más detalles ver Cuadro 10 y Figura 5.

Cuadro 10. Satisfacción en relación a trato. Fuente: Elaboración propia.

\begin{tabular}{|l|r|r|r|r|r|c|}
\hline $\begin{array}{l}\text { Nivel de } \\
\text { satisfacción }\end{array}$ & vacade & \multicolumn{1}{l}{ trares } & honcob & amable & higper & Prom. total \\
\hline 1 & 1,71 & 1,86 & 1,71 & 2,14 & 1,57 & 1,8 \\
\hline 2 & 2,42 & 2,42 & 2,71 & 2,37 & 2,47 & 2,48 \\
\hline 3 & 2,99 & 3,06 & 3,27 & 3 & 2,77 & 3,02 \\
\hline 4 & 3,67 & 3,78 & 4,07 & 3,69 & 3,51 & 3,74 \\
\hline 5 & 4,27 & 4,27 & 4,55 & 3,91 & 3,64 & 4,13 \\
\hline Satisfacción prom./Prom. total & 0,95 & 1,03 & 0,95 & 1,19 & 0,87 & 1,00 \\
\hline 1 & 0,98 & 0,98 & 1,09 & 0,96 & 0,99 & 1,00 \\
\hline 2 & 0,99 & 1,01 & 1,09 & 0,99 & 0,92 & 1,00 \\
\hline 3 & 0,98 & 1,01 & 1,09 & 0,99 & 0,94 & 1,00 \\
\hline 4 & 1,04 & 1,04 & 1,10 & 0,95 & 0,88 & 1,00 \\
\hline 5
\end{tabular}

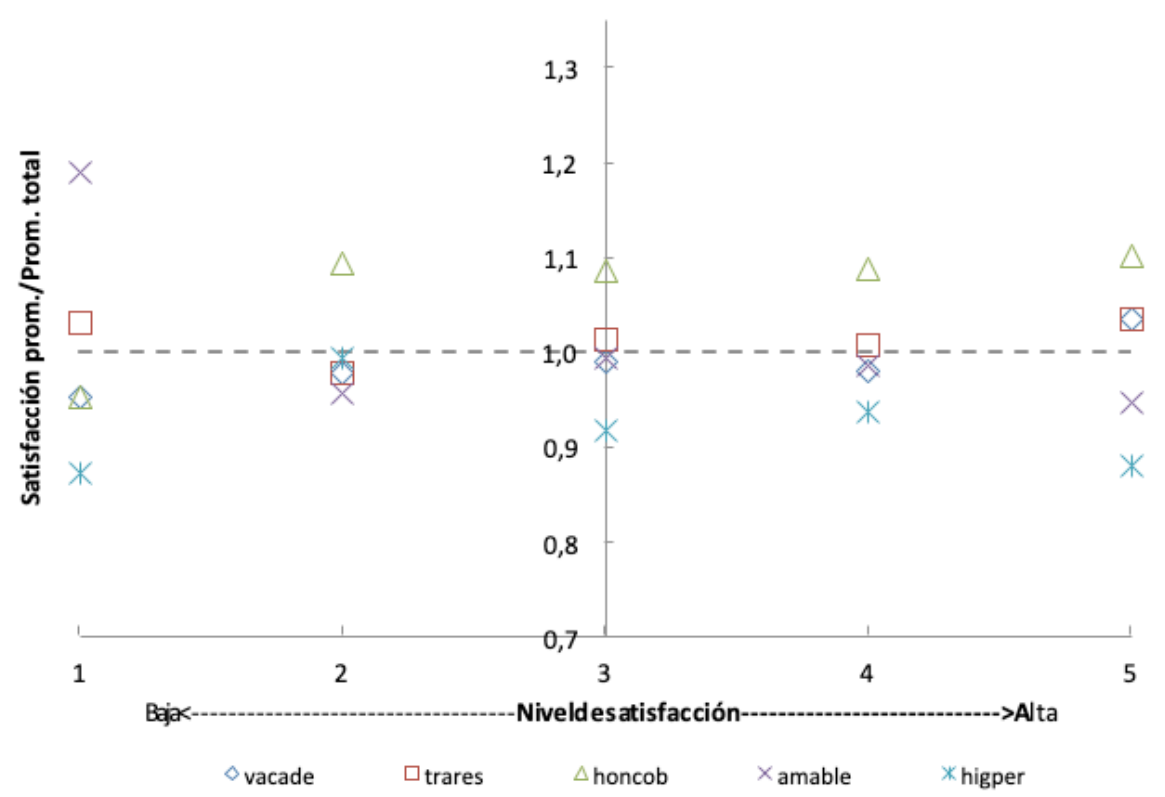

Figura 5. Satisfacción en relación a trato. Fuente: Elaboración propia.

Las especificaciones que explican en mayor porcentaje la satisfacción de edofís, en relación a la comodidad, fueron pasman y visven con valores entre $1 \%$ y $20 \%$; mientras la especificación asiade fue la que menor explica la valoración de la satisfacción del edofís con valores del 14\% y 2\% por debajo del promedio, ver Cuadro 11 y Figura 6. Una interpretación de estos resultados es que la existencia de visibilidad en las ventanas del autobús (visven) es la especificación de mayor peso, — desde el punto de vista del usuario- para emitir un juicio acerca de la satisfacción del estado físico del autobús 
(edofís) a partir de la comodidad; mientras que las condiciones adecuadas de los asientos (asiade) es la especificación que menor aportó para la valoración de la satisfacción del edofís (en relación a la comodidad). En el mismo tenor, la especificación espasi tiene mayor peso en la valoración de la satisfacción del edofís en comparación a la especificación asiade, un resultado lógico si se piensa que un usuario viajaría con una grado mayor de confort en un asiento "con aspecto sucio o maltratado" y dimensiones adecuadas que en un asiento "con aspecto limpio y buena apariencia" y dimensiones reducidas, que concuerda con el resultado presentado por dellólio et al. (2011) en que el confort durante el viaje tiene mayor peso que la limpieza interior del autobús para el sistema de pasajeros de la ciudad de Santander. El aporte entre 1\% y hasta de un 19\% que hace la visven se debe a que los usuarios se sienten con cierto nivel de confort y seguridad al poder constatar su ubicación en la ruta, y anticipar su bajada.

Cuadro 11. Satisfacción en relación a comodidad de edofis. Fuente: Elaboración propia.

\begin{tabular}{|l|c|l|l|l|l|l|}
\hline $\begin{array}{l}\text { Nivel de } \\
\text { satisfacción }\end{array}$ & pasman & \multicolumn{1}{l|}{ iliint } & espasi & asiade & visven & Prom. total \\
\hline 1 & 1,8 & 1,67 & 2 & 1,6 & 2,2 & 1,85 \\
\hline 2 & 2,47 & 2,39 & 2,38 & 2 & 2,41 & 2,33 \\
\hline 3 & 2,98 & 2,85 & 2,84 & 2,64 & 3,03 & 2,87 \\
\hline 4 & 3,6 & 3,53 & 3,59 & 3,43 & 3,62 & 3,55 \\
\hline 5 & 4,29 & 4,29 & 4,14 & 4,14 & 4,29 & 4,23 \\
\hline \multicolumn{7}{|l|}{ Satisfacción prom./Prom. total } \\
\hline 1 & 0,97 & 0,90 & 1,08 & 0,86 & 1,19 & 1,00 \\
\hline 2 & 1,06 & 1,03 & 1,02 & 0,86 & 1,03 & 1,00 \\
\hline 3 & 1,04 & 0,99 & 0,99 & 0,92 & 1,06 & 1,00 \\
\hline 4 & 1,01 & 0,99 & 1,01 & 0,97 & 1,02 & 1,00 \\
\hline 5 & 1,01 & 1,01 & 0,98 & 0,98 & 1,01 & 1,00 \\
\hline
\end{tabular}

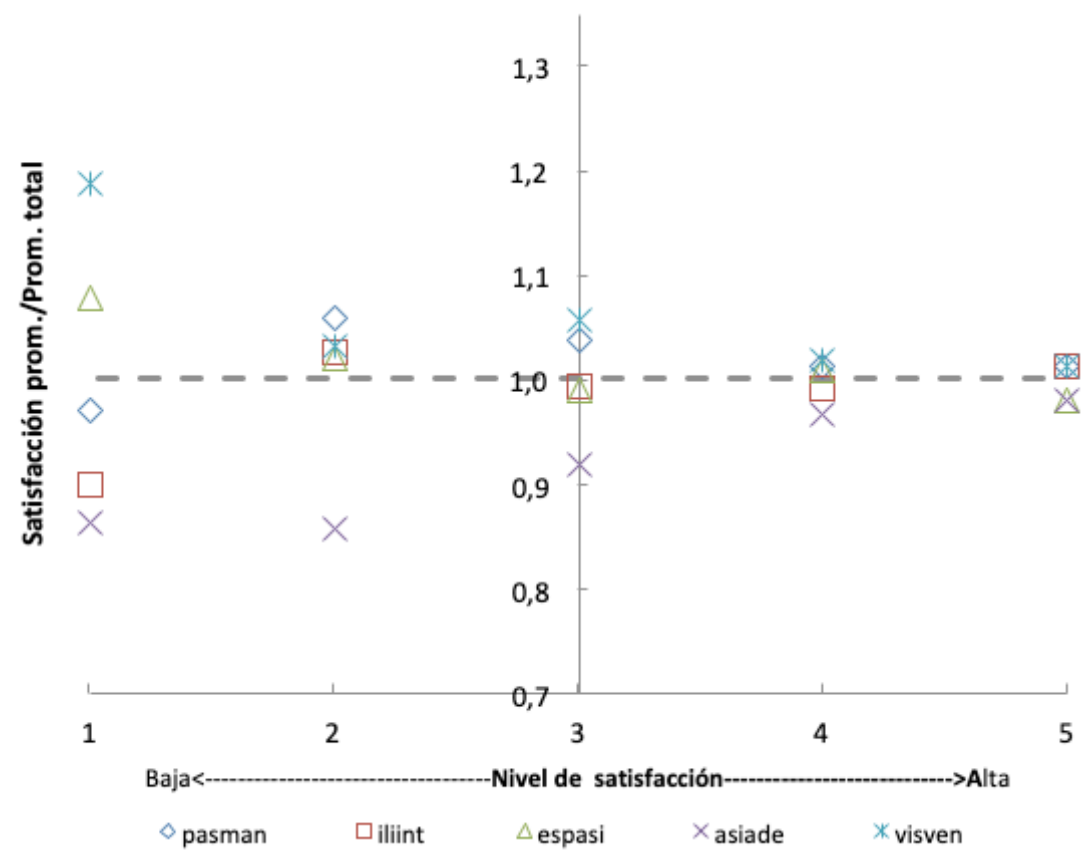

Figura 6. Satisfacción en relación a comodidad de edofís. Fuente: Elaboración propia. 
La especificación que explica la satisfacción del edofís, en relación a la seguridad (de sufrir un accidente), es pasman, con valores que oscilan el $2 \%$ y el $8 \%$, un resultado lógico si se piensa una vez que el usuario aborda el autobús lo primero que debe hacer -al menos para el caso de estudio- es sujetarse; la especificación que aporta más peso con valores entre 1 y $12 \%$ es la visven, es decir, el usuario del autobús con visibilidad en las ventanas se siente con mayor control del viaje (e.g. visualizar algunos sucesos en el exterior del autobús como las condiciones del tráfico o la maniobra de ascenso y descenso de pasajeros), es decir el usuario ostenta una mayor observación acerca de las condiciones (ambiente) durante del viaje en exterior del autobús (y no sólo en el interior), ver Cuadro 12 y Figura 7. Cabe mencionar que surge una relación entre el confort y la seguridad durante el viaje si se considera que a menor visibilidad (vidrios opacos o cortinas en ventanillas) hacia el interior del vehículo, el usuario podría percibir un mayor riego de algún tipo de crimen a bordo del autobús. Esta relación no es abarcada en este documento, pero existe evidencia de lo anterior para el caso de un sistema BRT para el caso de cinco ciudades latinoamericanas (Allen, et al. 2019).

Cuadro12. Satisfacción en relación a seguridad de edofís. Fuente: Elaboración propia.

\begin{tabular}{|c|c|c|c|c|c|c|}
\hline Nivel de satisfacción & pasman & iliint & espasi & asiade & visven & $\begin{array}{l}\text { Prom. } \\
\text { total }\end{array}$ \\
\hline 1 & 1,6 & 1,3 & 2,1 & 1,8 & 1,9 & 1,73 \\
\hline 2 & 2,4 & 2,2 & 2,4 & 2,0 & 2,2 & 2,26 \\
\hline 3 & 2,9 & 2,7 & 2,8 & 2,8 & 3,0 & 2,84 \\
\hline 4 & 3,6 & 3,5 & 3,5 & 3,4 & 3,6 & 3,52 \\
\hline 5 & 4,4 & 4,3 & 4,1 & 4,1 & 4,3 & 4,26 \\
\hline \multicolumn{7}{|c|}{ Satisfacción prom./Prom. total } \\
\hline 1 & 0,92 & 0,73 & 1,19 & 1,04 & 1,12 & 1,00 \\
\hline 2 & 1,08 & 0,98 & 1,08 & 0,88 & 0,98 & 1,00 \\
\hline 3 & 1,03 & 0,95 & 1,00 & 0,97 & 1,06 & 1,00 \\
\hline 4 & 1,02 & 0,99 & 0,99 & 0,98 & 1,03 & 1,00 \\
\hline 5 & 1,04 & 1,01 & 0,97 & 0,97 & 1,01 & 1,00 \\
\hline
\end{tabular}

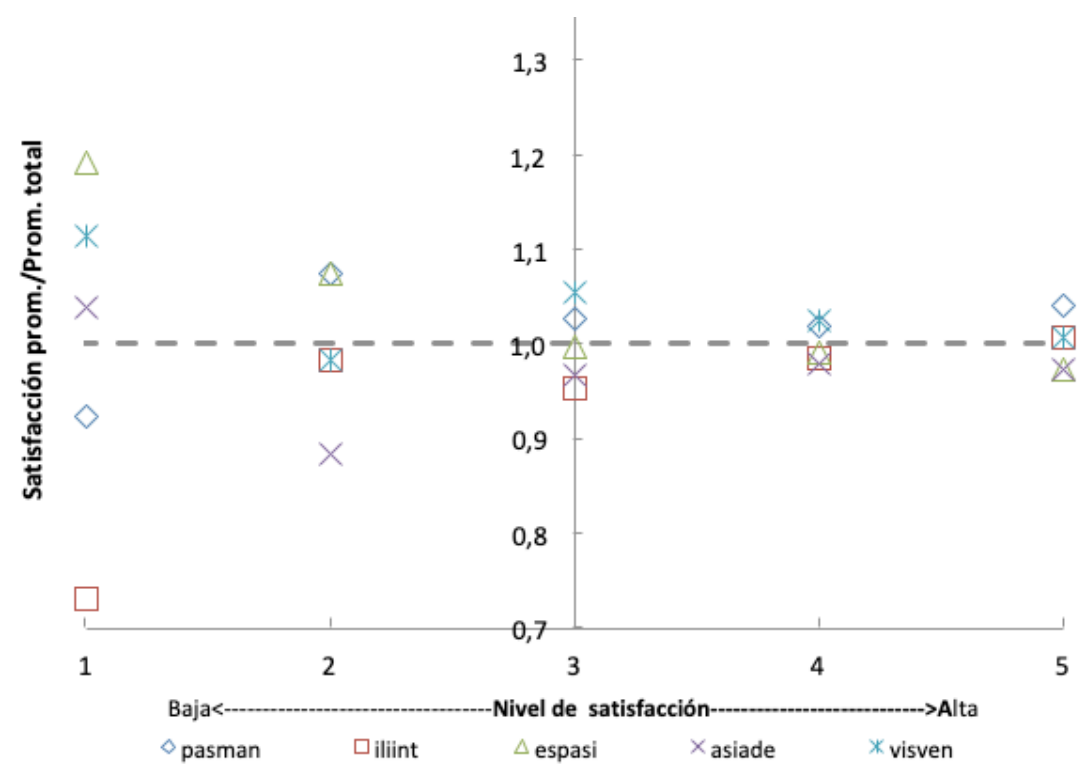

Figura 7. Satisfacción en relación a seguridad de edofis. Fuente: Elaboración propia. 
Considerando el juicio global de la satisfacción, el juicio de los entrevistados promedio fue de 2,8, el factor con mayor valoración fue el trato con 3,1, seguido del edofís con 3,0 ; en segundo lugar estuvo foma con 2,8 , mientras que el te, tv y $\mathrm{cv}$ fueron valorados similarmente, 2,4. Una interpretación de lo anterior indica que para tener un mayor impacto en la CS de autobuses de pasajeros de la zona de estudio se debe llevar a cabo implementaciones o mejorar en aspectos cualitativos, en este caso al estado físico del autobús, al trato al usuario y a la forma de manejar, más que en reducciones del tiempo o la tarifa, ver Cuadro 13 y Figura 8.

Cuadro13. Satisfacción global. Fuente: Elaboración propia.

\begin{tabular}{|l|r|r|r|r|r|r|r|}
\hline $\begin{array}{l}\text { Nivel de } \\
\text { satisfacción }\end{array}$ & \multicolumn{1}{l|}{ te } & \multicolumn{1}{l}{ tv } & \multicolumn{1}{l}{ cv } & \multicolumn{1}{l|}{ foma } & \multicolumn{1}{l|}{ trato } & edofís & $\begin{array}{l}\text { prom. } \\
\text { global }\end{array}$ \\
\hline 1 & 2,1 & 2,1 & 2,1 & 1,6 & 2,1 & 1,7 & 1,9 \\
\hline 2 & 2,3 & 2,3 & 2,3 & 2,4 & 2,8 & 2,4 & 2,4 \\
\hline 3 & 2,4 & 2,3 & 2,4 & 2,8 & 3,0 & 3,1 & 2,7 \\
\hline 4 & 2,6 & 2,6 & 2,7 & 3,4 & 3,7 & 3,7 & 3,1 \\
\hline 5 & 3,5 & 3,0 & 3,2 & 4,8 & 4,5 & 4,5 & 3,9 \\
\hline
\end{tabular}

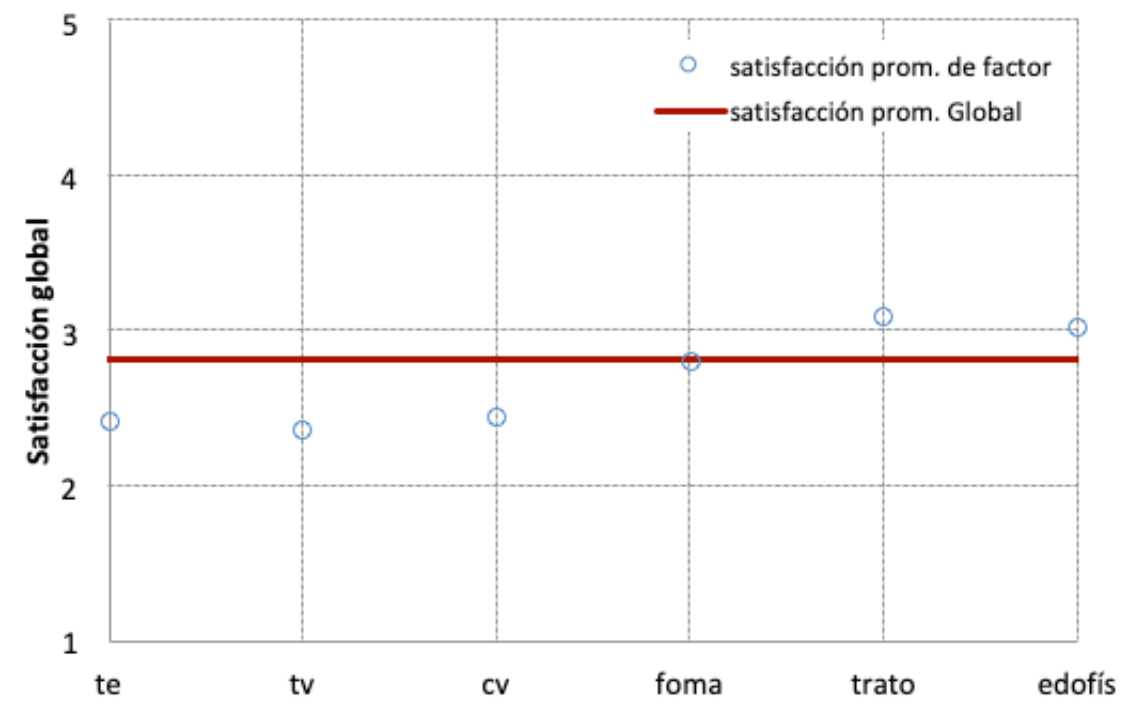

Figura 8. Satisfacción global. Fuente: Elaboración propia.

Se encontró que la sinuosidad de las rutas determina los tiempos y costos de transporte de pasajeros, lo que a su vez se refleja en los niveles de satisfacción de los usuarios. Adicionalmente la sobreposición de rutas, lo que se traduce en intervalos reducidos de paso en ciertos tramos de la red de transporte, no es un indicador que aumente los percepciones de satisfacción de los usuarios, debido a que los tiempos de espera son altos porque el usuario debe esperar el específico autobús que lo lleve a su destino. Es decir, el rediseño de la red de transporte de autobús más eficiente, reduciendo tiempos, trasbordos y costos del viaje, impactaría positivamente para cubrir las necesidades de movilidad de los usuarios.

En este documento se mostró una caracterización de la satisfacción del usuario del TP, lo que permite brindar a las empresas ofertantes del servicio y los agentes regulatorios mecanismos, que permitan, respectivamente, indicar en qué se debe mejor el servicio en concordancia con los deseos del usuario, y definir las políticas de TP a nivel táctico y 
operativo. La exposición presentada enriquece a ambas entidades acerca de la definición de la satisfacción, en particular brinda de ésta una visión particular y agregada de las necesidades del usuario, representadas en los elementos o factores que la integran, lo que sería posible detallar en contrato entre ofertante y regulador. El aporte no sólo es ofrecido a nivel global de factores, también presenta un análisis desmenuzado al interior de tres factores cualitativos, lo que permitiría a los administradores del transporte de pasajeros específicamente atender factores operativos; adicionalmente este estudio disminuye el hueco existente en la definición de factores intangibles. 


\section{Q Bibliografía}

»Allen, J., Muñoz, J. y Ortúzar, J. (2019) Understanding public transport satisfaction: Using Maslow's hierarchy of (transit) needs. Transport Policy, 81, pp. 75-94. https://doi.org/10.1016/j.tranpol.2019.06.005.

"Cárdenas, E. (2001) Características de la demanda de transporte en la Zona Metropolitana de la Ciudad de Toluca. Toluca: UAEM.

» de Oña, J. y de Oña, R. (2014). Quality of Service in Public Transport Based on Customer Satisfaction Surveys: A Review and Assessment of Methodological Approaches. Transportation Science, 48(1), pp. 1-18. DOI: 10.1287/trsc.2014.0544.

» dell'Olio, L., Ibeas, A. y Cecin, P. (2011) The quality of service desired by transport users. Transport Policy, 18(1), pp. 217-227. https://doi.org/10.1016/j. tranpol.2010.08.005.

»Eboli, L. y Mazzulla, G. (2009). A new customer satisfaction index for evaluating transit service quality. Journal of Public Transportation, 12(3), pp. 21-37. http://doi. org/10.5038/2375-0901.12.3.2.

»Elejabarrieta, F. y Iñiguez, L. (1984). Construcción de escalas de actitud tipo Thurst y Likert. <http://www.ict.edu.mx/acervo_bibliotecologia_escalas_ escalas\%2olikert-thust.pdf>.

» Gilbert, G. R. y Veloutsou, C. (2006). A cross-industry comparison of customer satisfaction. Journal of Services Marketing, pp. 298-308. https://doi. org/10.1108/08876040610679918.

" Grigoroudis, E. y Siskos, Y., (2010). Customer Satisfaction evaluation Methods for measuring and implementing service quality. New York: Springer.

» Hinojosa, R., Jiménez, P. L. y Campos, J. (2014). Transporte y calidad de vida en el Área Metropolitana de Toluca. En: Encuentro Interno Transdisciplinario de los sistemas de Información y Tecnología en las ciencias Nezahualcóyotl, México.

"Losby, J. y Wetmore, A. (2012). Using Likert Scales in evaluation Survey Work. Atlanta: Centers for Disease Control and Prevention. https://www.cdc.gov/ dhdsp/pubs/docs/CB_February_14_2012.pdf.

»Parasuraman, A., Zeithaml, V. y Berry, L. (1985). A conceptual model of service quality and its implication for future research. Journal of Marketing, 49, pp. 4150. DOI: $10.2307 / 1251430$.

»Romero, J., Gaspar, N. y Bautista, E. (2016). Percepción de la satisfacción del servicio en el transporte público solo para mujeres. Revista Transporte y Territorio, 15, pp. 164-182. http://revistascientificas.filo.uba.ar/index.php/rtt/ issue/view/181.

» Romero, J., Gaspar, N., Torres, R.y López, R. (2017). El transporte público y privado en relación a la periferia de la ciudad de Toluca. Diotima, Revista Científica de Estudios Transdisciplinaria, 2(5), pp. 35-45. http://www.revista-diotima.org/.

"Stopher, P. (2012). Collecting, Managing, and Assessing Data Using Sample Surveys. Primera Edición ed. Cambridge: Cambridge University Press.

»UAEM-GEM (2016). Programa de movilidad del Estado de México, Universidad Autónoma del Estado de México-Gobierno del Estado de México. 
» van de Velde, D. (1999). Organisational forms and entrepreneurship in public transport Part 1: classifying organisational forms. Transport Policy, 6, 47-157. DOI: 10.1016/Sog67-070X(99)ooo16-5.

\section{Javier Romero-Torres / jromero@uaemex.mx}

Candidato a Doctor en Ingeniería en Transporte por la Universidad Autónoma del Estado de México. Actualmente es Profesor de Tiempo Completo en el Centro Universitario UAEM Nezahualcóyotl de la misma casa de estudios. Sus áreas de investigación comprenden temas en transporte público, calidad de servicio, ingeniería de tránsito y en modelos de elección discreta, $y$ ha asistido regularmente a los principales foros académicos y científicos del área de movilidad y transporte en México y Latinoamérica.

\section{Javier García-Gutiérrez / jgarciag@uaemex.mx}

Investigador en la Facultad de Ingeniería de la Universidad Autónoma del Estado de México. Tópicos de investigación: modelado de ingeniería de cadena de suministro, optimización de procesos multi-objetivos y problemas combinatorios.

\section{Noé Gaspar Sánchez / noe-urbanismo@outlook.com}

Maestro en Estudios Urbanos y Regionales y Doctor en Urbanismo. Profesor de Tiempo Completo en el Centro Universitario UAEM Nezahualcóyotl de la Universidad Autónoma del Estado de México. Líneas de Investigación: Movilidad Urbana y Procesos Metropolitanos. Publicaciones en revistas internacionales: Revista Proyección- CIFOT de la Universidad Nacional de Cuyo, Mendoza Argentina; Revista Urbano de la Universidad de Bío-Bío de Chile y Bitácora Urbana Territorial de la Universidad Nacional de Colombia. 